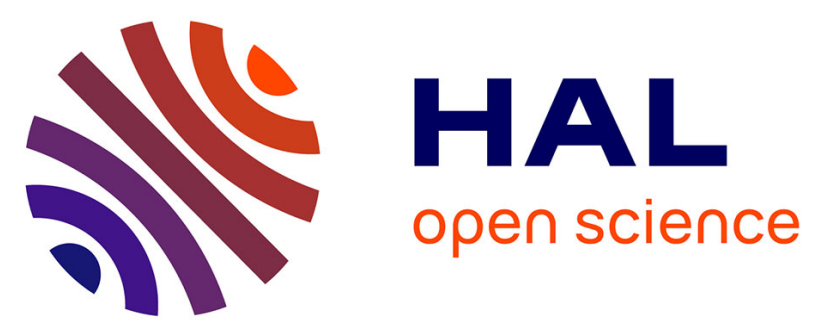

\title{
MODELING ULTRASONIC WAVE PROPAGATION IN A DENTAL IMPLANT - BONE SYSTEM
}

\author{
A Dorogoy, Guillaume Haiat, K Shemtov-Yona, D. Rittel
}

\section{To cite this version:}

A Dorogoy, Guillaume Haiat, K Shemtov-Yona, D. Rittel. MODELING ULTRASONIC WAVE PROPAGATION IN A DENTAL IMPLANT - BONE SYSTEM. Journal of the mechanical behavior of biomedical materials, 2020, 103, pp.103547. hal-02387481

\section{HAL Id: hal-02387481 https://hal.science/hal-02387481}

Submitted on 29 Nov 2019

HAL is a multi-disciplinary open access archive for the deposit and dissemination of scientific research documents, whether they are published or not. The documents may come from teaching and research institutions in France or abroad, or from public or private research centers.
L'archive ouverte pluridisciplinaire HAL, est destinée au dépôt et à la diffusion de documents scientifiques de niveau recherche, publiés ou non, émanant des établissements d'enseignement et de recherche français ou étrangers, des laboratoires publics ou privés. 
MODELING ULTRASONIC WAVE PROPAGATION IN A DENTAL IMPLANT BONE SYSTEM

\author{
A. Dorogoy ${ }^{1}$, G. Haiat ${ }^{2}$, K. Shemtov-Yona ${ }^{1}$ and D. Rittel ${ }^{1 *}$ \\ ${ }^{1}$ Faculty of Mechanical Engineering, Technion, 32000, Haifa, Israel \\ ${ }^{2}$ CNRS, Laboratoire Modélisation et Simulation Multi-échelle, UMR CNRS 8202, 94010 Créteil Cedex, \\ France
}

\title{
Keywords
}

Dental implants, ultrasound, bone-implant interface, finite element, waves, stability

* Corresponding author: merittel@technion.ac.il 


\section{ABSTRACT}

The evolution of the bone-implant interface reflects the implant osseointegration and bond strength, thereby determining the overall implant stability in the jawbone. Quantitative ultrasound represents a promising alternative technique to characterize the interfacial integrity, precisely due to the fact that those waves propagate essentially along the bone-implant interface, and are therefore influenced by its state. This study reports a numerical investigation of ultrasonic wave propagation for a commercial implant-jawbone system in which the thickness and mechanical properties of the interfacial layer (corresponding to the interphase) are systematically varied through the application of a rule of mixtures, in order to mimic the evolution from a dominantly soft tissue - like medium up to a fully healed bone.

A simple figure of merit is devised in terms of an RMS-like (root mean square) factor based on the implant displacements, that evolves continuously and significantly with the bone "healing" process, thereby providing unequivocal information on the nature of the investigated bone-implant interface.

The results show that the wave propagation pattern is primarily dictated by the impedance mismatch rather than by the interface thickness. This study validates the concept of quantitative ultrasonic testing as a sensitive alternative to the widespread resonant frequency analysis, thereby opening the way for future sensitivity analyses that will address more refined bone-implant interface pathologies such as those observed in the clinical realm. 


\section{Introduction}

The long-term success of dental implants depends on a long-lasting osseointegration and stable contact with the peri-implant bone. Osseointegration and implant stability depend on the quality of the surrounding bone and on the biomechanical properties of the bone-implant interface (BII) (Albrektsson, 2008). Today, long-term prognosis regarding implants is based on stability measurements. The evolution of the biomechanical properties of the bone-implant interface (BII), remains quite difficult to monitor in vivo by non-destructive methods. Yet, implant stability can be quantitatively assessed through non-invasive techniques, discussed in the sequel, that can be part of the clinical environment (Gao et al., 2019; von Wilmowsky et al., 2014; Zanetti et al., 2018). Despite their clear advantage as clinically easy to use devices, their reliability and correlation to the bone-implant properties remains to be established (Aparicio et al., 2006; Atieh et al., 2012, 2014; Manresa et al., 2014; Nkenke et al., 2003; Zanetti et al., 2018).

Investigating of the biomechanical properties of the bone-implant interface can be done using quantitative ultrasound (QUS)(De Almeida et al., 2007). The principle of the measurement relies on the dependence of ultrasonic wave propagation within the implant on the bone properties around the bone-implant interface (Mathieu et al., 2011b). The principle of the measurement was validated experimentally by showing the sensitivity of the echographic response of a planar BII to healing time using a coin-shaped implant model (Mathieu et al., 2012). Significant variations of the ultrasonic response of dental implants embedded in a bone substitute biomaterial were shown to occur when the implants are subjected to fatigue loading (Vayron et al., 2013). Other in vitro studies proved the potentiality of QUS methods to assess the primary stability of dental implants inserted in bone phantom (Vayron et al., 2018a) and in bovine bone tissue (Vayron et al., 2014a). A preclinical validation of the device in rabbits (Vayron et al., 2014b) and in sheep (Vayron et al., 2018b) was carried out and showed that i) the measurement was sensitive to healing time and ii) a significant correlation of the measurement with the bone-implant contact (BIC) ratio measured with histology. A finite element model was developed at the microscopic level to account for the effect of the surface roughness and of osseointegration phenomena of a planar bone-implant interface (Hériveaux et al., 2018). At the macroscopic level, the propagation of ultrasound in cylindrical implants was simulated using finite difference time domain simulation (Mathieu et al., 2011a) and finite element numerical simulations (Vayron et al., 2015), leading to a better performance of the device. Then, a 2-D axisymmetric finite element model was used to model the interaction between a dental implant and an ultrasonic wave considering a realistic implant geometry (Vayron et al., 
2016). However, each bone properties were varied independently (Vayron et al., 2016) while they all vary in parallel in clinical practice and it still remains difficult to determine the influence of the biomechanical properties corresponding to osseointegration phenomena and of the thickness of a bone layer surrounding an implant on its ultrasonic response.

In this work we consider a single generic implant geometry that is fully inserted in a jawbone section made of cortical and trabecular bone. A peri-implant bone layer is assigned with various stiffness values and widths to mimic the evolution of the peri-implant bone healing. The periimplant layer follows the geometry of the threaded implant. The implant is assumed to be fully bonded to the bone. The aim of this paper is to characterize the ultrasonic response of the dental implant using a finite element model for various kinds of peri-implant layer conditions.

The novelty of this investigation is in the consideration of a more realistic bone geometry with cortical bone tissue located all around the implant. It also considers the influence of the thickness of the peri-implant bone layer where osseointegration occur. This thickness is an important parameter for the implant success and in this work it follows the implant threads geometry which is more realistic. Moreover, the combined variation of the Young's modulus, Poisson's ratio and the mass density during the healing process is considered. A better indicator was defined and successfully used to characterize and quantify the vertical displacements of the upper face of the peg due to ultrasound excitation.

The usage of finite element modeling is mandatory in order to understand the influence of osseointegration on the ultrasonic response of an implant because the variation of the different bone properties is difficult to control experimentally.

\section{Material and methods}

\subsection{Geometry and Assembly}

An 2D axisymmetric model is used in the analyses, as assumed in (Vayron et al., 2016). The axisymmetric model was derived from a 3D model of the mandible bone and implant shown in Fig.1a,b, similarly as in (Dorogoy et al., 2017; Rittel et al., 2017). The 3D model is shown in Fig. 1a, while a side view from which the axisymmetric model was derived is shown in Fig $1 \mathrm{~b}$, and detailed in Fig 1c. 
The assembled axisymmetric model is comprised of 2 parts:

1) A mandible bone (Fig. 1d)

2) A dental implant bonded to an extension, subsequently referred to as "peg" (Fig. 1e).

The bone consists of an outer cortical bone (grey color in Fig 1c,d) having a thickness of $\sim 2 \mathrm{~mm}$, and of inner cancellous/trabecular bone (cream color in Fig. 1c,d). The implant and peg (Fig. 1e) are made of a commonly used titanium alloy (Ti6Al4V).

An axisymmetric model was used because a very fine mesh is required to model ultrasonic wave propagation (Drozdz, 2008; Egerton et al., 2017) as discussed below, so that this model reduces significantly the computational size of the problem, as opposed to a prohibitively large full 3D model. Alternatively, a 2D model (e.g. plane strain) would grossly distort the geometry of the implant. The assumption of axial symmetry has a minor effect on the results as long as the reflecting pulses reaching the top of the peg are originating from the bone-implant interface (BII), the surrounding peri-implant layer and the trabecular bone. The model will lose its similarity to the real 3D bone at longer times for which reflections of shear wave from the cortical bone reach the top of the peg (See section 3.2 and Fig.2 in the sequel).

Perfect bonding was prescribed between all contacting materials. It was assumed that a peri-implant bone layer is affected (damaged) by the implant insertion process. This weakened layer has an axisymmetric shape which follows the implant threads' shape as shown in Figs. 1f,g. The width $w$ of this peri-implant layer was assumed to be $w=0.1 \mathrm{~mm}$ (Fig. 1f) and $w=0.2 \mathrm{~mm}$ (Fig. 1g), respectively. 


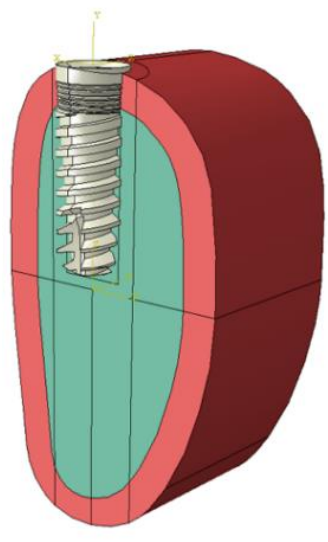

a.

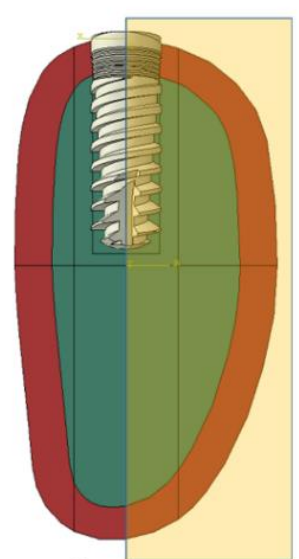

b.

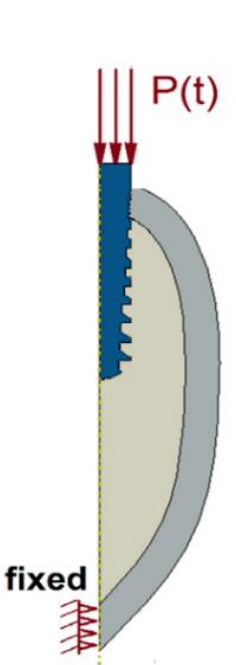

c.

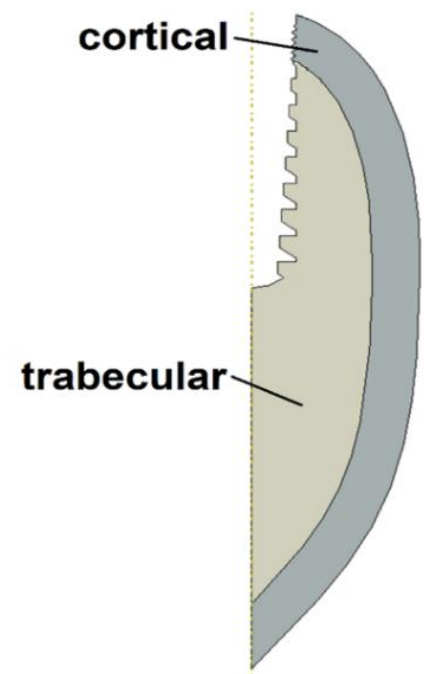

d.

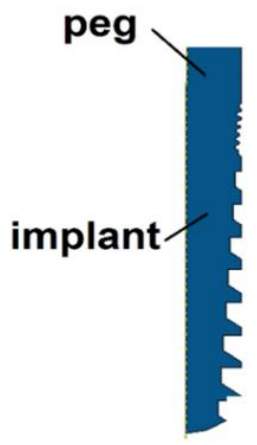

e.

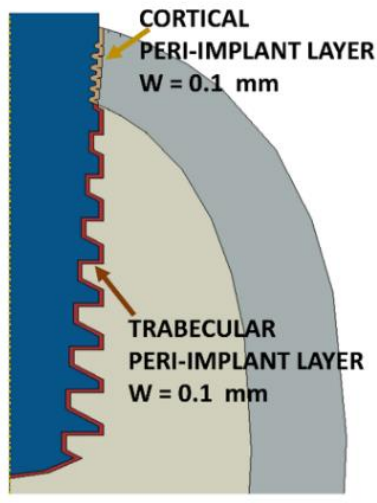

f.

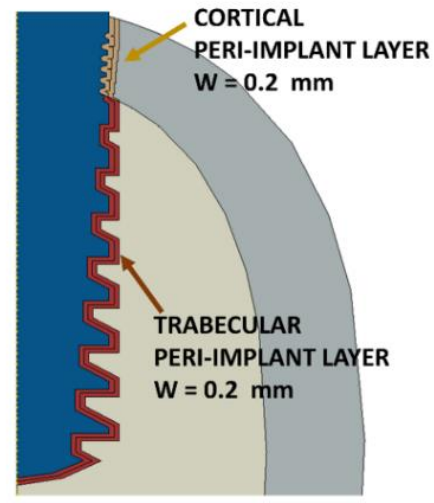

g.

Figure 1: a. Isometric view of exposed jaw bone with a dental implant. b. Side view of exposed jaw bone with a dental implant. c. The axisymmetric assembly. d. The mandible bone. e. The dental implant. f. Detail showing a peri-implant weakened layers of $0.1 \mathrm{~mm}$ wide. g. Detail showing a $0.2 \mathrm{~mm}$ wide peri-implant weakened layer. 


\subsection{Boundary conditions}

A pressure $\mathrm{P}(\mathrm{t})$ is applied at the peg top face (Fig. 1c) and writes (Vayron et al., 2016a):

$$
P(t)=P_{0} \sin (2 \pi f t) \cdot e^{-4(f t-1)^{2}}
$$

where $\mathrm{P}_{0}=\mathrm{F} / \mathrm{A}$, $\mathrm{A}$ is the area of the top peg face, and $\mathrm{F}=1 \mathrm{~N}$, which is chosen arbitrarily since the problem is fully linear. The frequency is $f=10 \mathrm{MHz}$. The amplitude is set to 0 for $\mathrm{t}>2 \mu \mathrm{s}$.

The assembly is fixed at the bottom of the bone. Fully constrained ("encastre") conditions are applied at the center of the cortical bone (Fig. 1c).

\subsection{Materials}

For the sake of simplicity, all three parts of the model were assigned linear elastic and homogenous material properties. For the implant and peg, isotropic mechanical properties of Ti-6Al-4V ELI (American Society for Testing and and American Society for Testing and Materials, 2013) were used (see Table 1). Cortical and trabecular bone tissues were assumed to be isotropic with mechanical properties chosen according to (Vayron et al., 2015b) (see Table 1, $\xi=0$ ).

It is assumed that immediately after the insertion, the peri-implant layer is made mostly of blood or soft tissues, for which water (liquid) properties are assumed for the sake of simplicity. During the healing process the layer becomes mineralized as a result of the osseointegration process. After a long time (typically several months), the properties of the peri-implant layer become identical to those of the host bone. A parameter $\xi$ represents the amount of water in the mixture, $0 \leq \xi \leq 1$. For $\xi=1$ (respectively $\xi=0$ ), the mixture properties are those of water (respectively the host bone). The Young's modulus (E), the Poisson's ratio ( $v)$ and the density $(\rho)$, which are the input parameters for the finite element calculations, were assigned values according to Eqns. 2-4 (rule of mixtures).

$$
\begin{aligned}
& E(\xi)=E_{\text {water }} \xi+E_{\text {bone }}(1-\xi) \\
& v(\xi)=v_{\text {water }} \xi+v_{\text {bone }}(1-\xi) \\
& \rho(\xi)=\rho_{\text {water }} \xi+\rho_{\text {bone }}(1-\xi)
\end{aligned}
$$

The Lamé coefficients $(\lambda)$ and $(\mu)$, the shear modulus, are determined according to Eqns. 5-6: 


$$
\begin{aligned}
\lambda(\xi) & =\frac{E(\xi) v(\xi)}{(1+v(\xi))(1-2 v(\xi))} \\
\mu(\xi) & =\frac{E(\xi)}{2(1+v(\xi))}
\end{aligned}
$$

The longitudinal wave velocity $\left(C_{L}\right)$ and the shear wave velocity can be determined for each composition (mixture) according to Eqns. 7-8.

$$
\begin{aligned}
& C_{L}(\xi)=\sqrt{\frac{\lambda(\xi)+2 \mu(\xi)}{\rho(\xi)}} \\
& C_{S}(\xi)=\sqrt{\frac{\mu(\xi)}{\rho(\xi)}}
\end{aligned}
$$

Throughout this work, we considered $0.0 \leq \xi \leq 0.9$, rather than 1 to avoid modeling a pure water phase.

Table 1: Material properties. The peri-implant layers properties are determined according to the rule of mixtures (Eqns. 2-4).

\begin{tabular}{|c|c|c|c|c|c|c|}
\hline & $\xi$ & $E[G P a]$ & $v$ & $\rho\left[\mathrm{Kg} / \mathrm{m}^{3}\right]$ & $\lambda[G P a]$ & $\mu[\mathrm{GPa}]$ \\
\hline water* & 1.0 & 0.0002 & 0.5 & 1000 & 2.25 & 0.0001 \\
\hline $\begin{array}{c}\text { Ti-6Al-4V } \\
\text { ELI** }\end{array}$ & - & 113.8 & 0.33 & 4430 & 83.05 & 42.78 \\
\hline cortical & $0.0 *$ & 16.45 & 0.37 & 1850 & 17.6 & 5.99 \\
\cline { 2 - 7 } & 0.3 & 11.51 & 0.41 & 1595 & 18.87 & 4.08 \\
\cline { 2 - 7 } & 0.5 & 8.22 & 0.44 & 1425 & 19.68 & 2.86 \\
\cline { 2 - 7 } & 0.6 & 6.58 & 0.45 & 1340 & 20.07 & 2.27 \\
\cline { 2 - 7 } & 0.7 & 4.93 & 0.46 & 1255 & 20.46 & 1.69 \\
\cline { 2 - 7 } & 0.8 & 3.29 & 0.47 & 1170 & 20.84 & 1.12 \\
\cline { 2 - 7 } & 0.9 & 1.65 & 0.49 & 1085 & 21.22 & 0.55 \\
\hline trabecular & $0.0 *$ & 1.7 & 0.3 & 1170 & 1.28 & 0.85 \\
\cline { 2 - 7 } & 0.3 & 1.19 & 0.36 & 1119 & 1.13 & 0.44 \\
\cline { 2 - 7 } & 0.5 & 0.85 & 0.40 & 1085 & 1.21 & 0.30 \\
\cline { 2 - 7 } & 0.6 & 0.68 & 0.42 & 1068 & 1.26 & 0.24 \\
\cline { 2 - 7 } & 0.7 & 0.51 & 0.44 & 1051 & 1.30 & 0.18 \\
\cline { 2 - 7 } & 0.8 & 0.34 & 0.46 & 1034 & 1.34 & 0.12 \\
\cline { 2 - 7 } & 0.9 & 0.17 & 0.48 & 1017 & 1.38 & 0.06 \\
\hline
\end{tabular}

* Vayron et al., 2015

** American Society for Testing and and American Society for Testing and Materials, 2013 


\subsection{Mesh}

The recommended mesh density for simulating the propagation of ultrasonic waves is (Egerton et al., 2017) :

$d x \leq \frac{\lambda_{0}}{20}=\frac{C_{S} / f}{20}$.

Where $\mathrm{dx}$ is the mesh size, $\lambda_{0}$ is wave length, $\mathrm{C}_{\mathrm{s}}$ is the shear wave velocity and $\mathrm{f}$ is the wave frequency. The mesh density is determined in terms of node spacing and not element side size (Drozdz, 2008). We used quadratic triangular elements of type CAX6M. It was proved that for a given mesh density, quadratic quadrilateral elements strongly reduce the velocity error (Drozdz, 2008). The quadratic elements which are used herein have mid-edge nodes and the distance between the nodes is estimated as half of the element side size. The smallest elements were applied on the trabecular peri-implant layer and have mesh density of $1.25 \mu \mathrm{m}$. Applying the recommended mesh density results in a mesh comprised of $\sim 4$ millions elements for $w=0.2 \mathrm{~mm}$ and $\sim 3$ millions elements for $w=0.1 \mathrm{~mm}$, respectively.

\section{Results}

The total time simulated duration was $15 \mu \mathrm{s}$. A total of 13 cases were solved with a mesh density of $\mathrm{dx}=\lambda_{0} / 20$ for values of $\xi=0.0,0.3,0.5,0.6,0.7,0.8$ and 0.9 , for each peri-implant layer's thickness (0.1 and $0.2 \mathrm{~mm})$.

The averaged vertical displacement at the top of the peg was monitored (numerically) during the first $15 \mu$ s from application of the ultrasonic pulse (Eqn. 1). Monitoring was done at $100 \mathrm{MHz}$, yielding 1500 equally spaced time points with $\Delta \mathrm{t}=0.01 \mu \mathrm{s}$.

\subsection{Wave propagation}

The Tresca stress variation for case $\mathrm{w}=0.1 \mathrm{~mm}$ and $\xi=0.5$ is shown for illustration in Fig. 2 for the time interval of $1 s \quad t \quad 8 \mathrm{~s}$. The axisymmetric model is rotated by $270^{\circ}$ to visualize the difference between the numerical model and a real bone which is shown in Figs. 1a,b. Note that the model proposed here is an approximation to the real model shown in Fig 1a,b. At time $0 s t 1 s$, the shear wave propagates mostly along the peg+implant and reaches the upper BII. At $1 s \quad t \quad 2 s$, some of the wave has started passing the BII. At $2 s t 8 s$, the wave 
has propagated along the whole BII and passed the tip of the implant. The Tresca stress spreads significantly within the trabecular bone. The wave propagates always faster within the cortical bone (axial) than within the trabecular bone (radial).
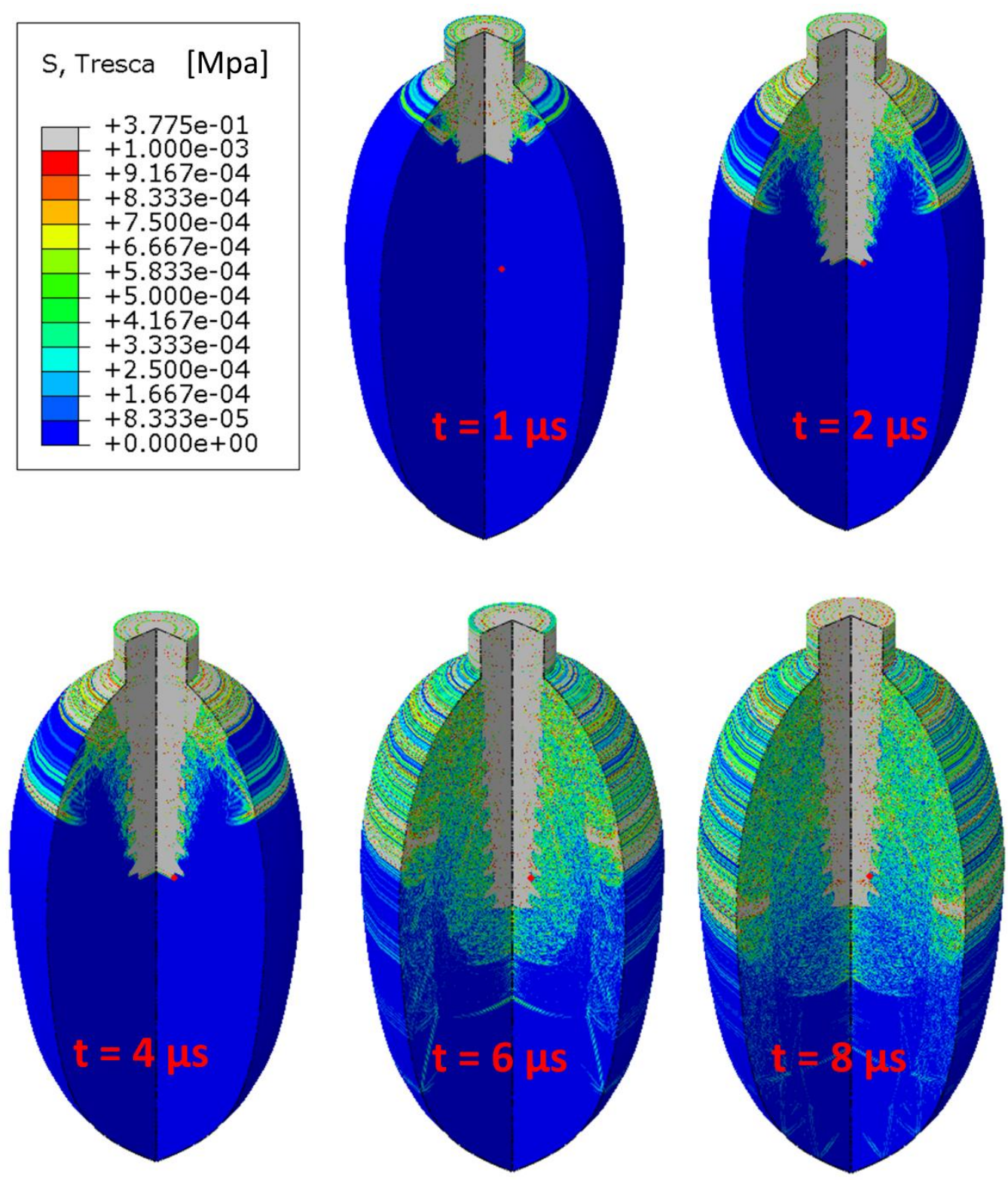

Figure 2: 3D visualization of the axisymmetric model at $1 \mu s \leq t \leq 8 \mu s$ showing the Tresca stress wave propagation.

\subsection{An indicator of the implant's ultrasonic response}

In order to better characterize, and quantify to some extent the vertical displacements of the upper face of the peg, a new indicator that bears some similarity with the RMS, was defined, as shown in Eq. (10): 
$I\left(t^{*}\right)=\int_{0}^{t^{*}}[f(t)]^{2} d t$

where $f(t)$ are the above-mentioned vertical displacements.

This indicator is not a constant, as would be calculated from an RMS estimate, but rather a function of time $\left(\mathrm{t}^{*}\right)$. The units of this indicator are $\left[\mathrm{mm}^{2} \mathrm{~s}\right]$ but they can be multiplied or normalized arbitrarily, hence we just mention values without units through the sequel.

Eqn. 10 is a modification of the indicator which was used by (for example) (Vayron et al., 2016b)

in which the function is not squared and can be interpreted as $I\left(t^{*}\right)=\int_{0}^{t^{*}} f(t) d t$. The squaring enhances the capability of characterization the effect of peri-implant properties.

\subsection{Top peg average vertical displacements}

Figure 3a shows the top peg average vertical displacement after application of the indicator (Eq. 9) for $0<t^{*}<15 \mu \mathrm{s}$. Solid (respectively dashed) lines correspond to results obtained with $w=0.1$ mm (respectively $w=0.2 \mathrm{~mm}$ ). Two regions can be distinguished in Fig. $3 \mathrm{a}: 0<\mathrm{t}^{*}<\mathrm{t}_{0}$ and $\mathrm{t}^{*}>$ $t_{0}$. Until $t^{*}=t_{0}$ there is no difference between all cases because the top peg displacements are due to the applied load and reflected waves from the peg. At $t^{*}=t_{0}$, waves which are reflected by the BII reach the top of the peg, hence differences between the cases start to be increasingly visible. As time increases, more waves are passing the BII and get to the top of the peg and the indicator values increase as well.

Figure $3 b$ shows the results with translation to the axis ( $\left.t^{\prime}, I^{\prime}\right)$ shown on Fig. $3 a$ :

$$
\begin{aligned}
& I^{\prime}\left(t^{*}-t_{0}\right)=\int_{0}^{t^{*}}[f(t)]^{2} d t-I_{0} \\
& I_{0} \equiv \int_{0}^{t_{0}}[f(t)]^{2} d t
\end{aligned}
$$




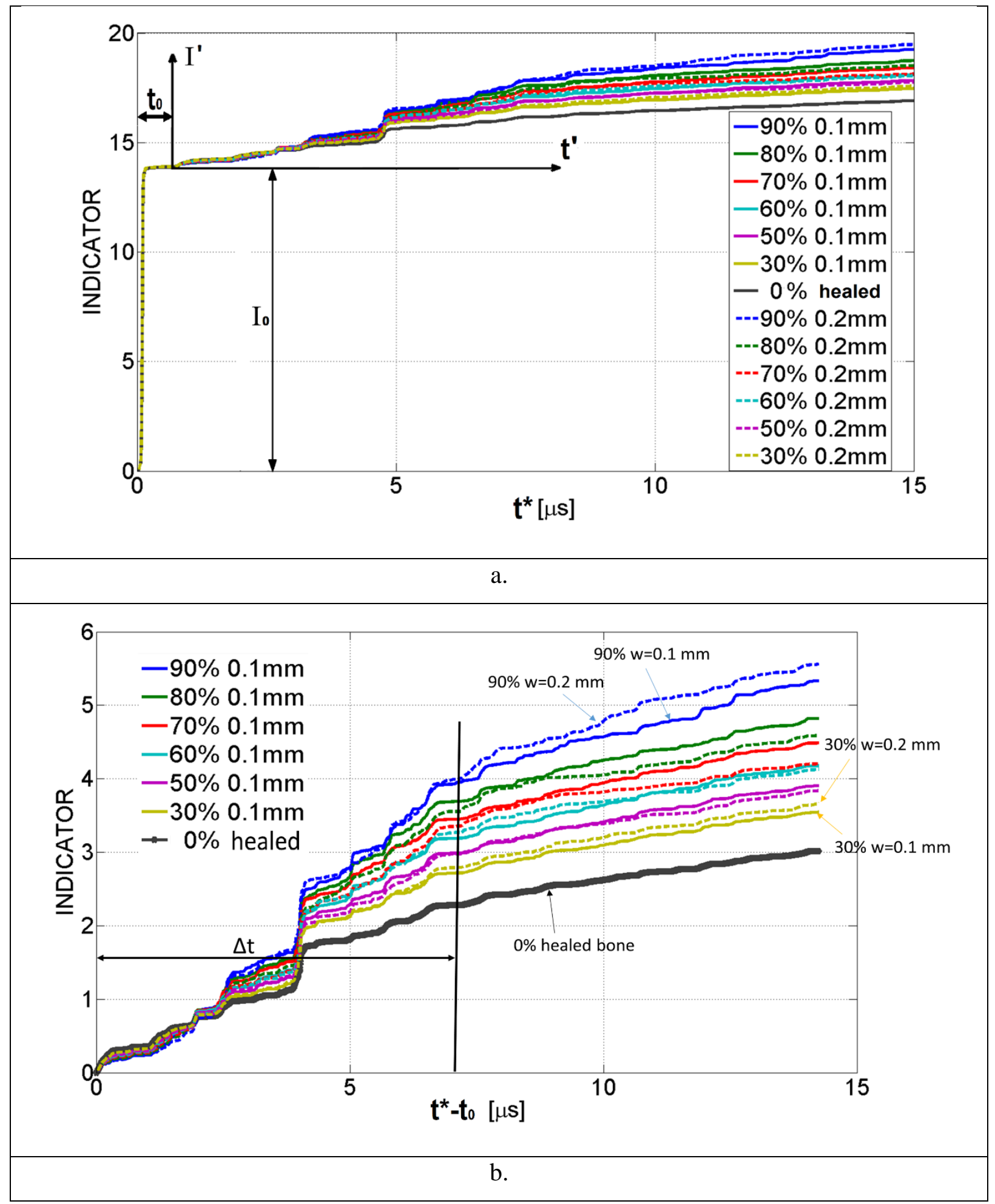

Figure 3: Indicator values applied to the top peg vertical displacements. a. The indicator Eqn. 9. b. The translated indicator values Eqns. 10-11.

The results shown in Fig. 3 show that the weaker the peri-implant layer, the higher the indicator value for all $\mathrm{t}^{*}$. At the same time, it appears that the two peri-implant layer thicknesses of 0.1 and $0.2 \mathrm{~mm}$ do not influence significantly the indicator's values. 
It can thus concluded that wave propagation is mostly dictated by the mechanical impedance mismatch at the BII and is just slightly affected by the peri-implant layer's thickness (impedance mismatch is the ratio between the multiplication of the density $\rho$ and wave velocity $\mathrm{C}$ on both sides of an interface: $\frac{\rho_{1} \cdot C_{1}}{\rho_{2} \cdot C_{2}}$ - the subscript 1,2 refer to sides of an interface. This ratio affects the relative reflection and transmission of a wave through a heterogeneous interface).

It should be noted that for $\mathrm{t}^{*}>\Delta \mathrm{t}(\Delta \mathrm{t}=7 \mu \mathrm{s})$, the recorded displacements at the top of the peg include reflections from the CTI (Cortical Trabecular interface) hence these displacements do not represent faithfully experimental displacements from a real bone (Fig. 1a,b) which does not possess radial symmetry. Nevertheless, the results indicate that longer pulses will increase the Indicator values and therefore its usability.

The indicator values at $\mathrm{t}^{*}=8 \mu \mathrm{s}$ and $\mathrm{t}^{*}=15 \mu \mathrm{s}$ are plotted against $\xi[\%]$ in Fig. 5. The markers represent numerical results while the lines are fit to these results. Solid lines are for $\mathrm{w}=0.1 \mathrm{~mm}$ and dash lines for $\mathrm{w}=0.2 \mathrm{~mm}$. Once again, it can be observed that the width of the damaged periimplant layer has a minor effect on the indicator values. The behaviour of the curves at $\mathrm{t}^{*}=8 \mu \mathrm{s}$ and $\mathrm{t}^{*}=15 \mu \mathrm{s}$ is similar. Two gradients $\beta_{1}$ and $\beta_{2}$ for $\xi>50 \%$ and $\xi<50 \%$ can be identified. Since $\beta_{1}>\beta_{2}$ it can be concluded that the indicator will be more sensitive in the early stages of healing while it might have a lower resolution as the healing process progresses. Nevertheless, the differences in the indicator values at each time between a fully healed and fully deteriorated bone is large (150-200\%) - this fact indicate that measurements of US waves can be used to identify healing.

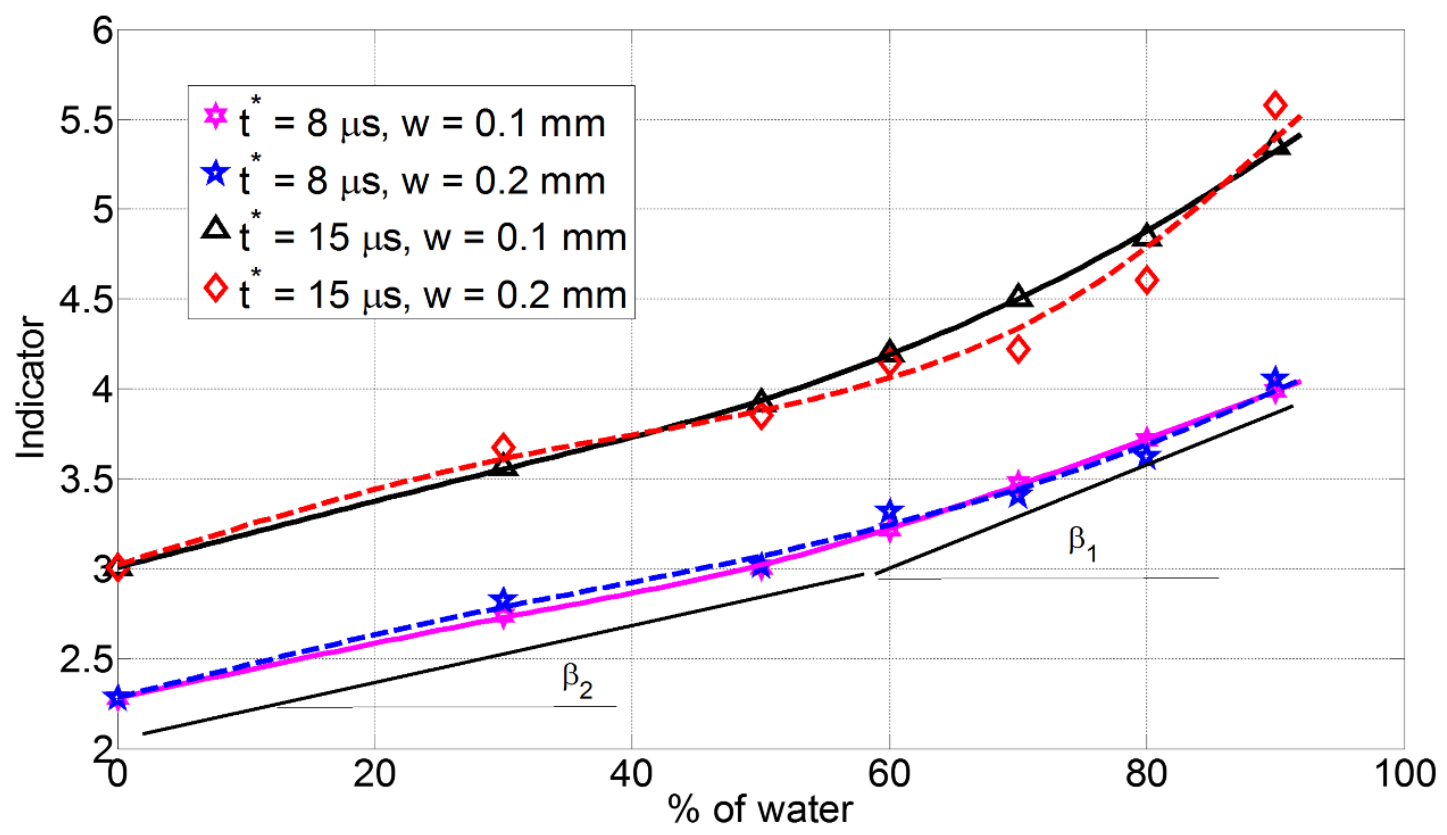


Figure 4: The indicator values at $8 \mu$ s and $15 \mu$ s versus the healing process $(\xi)$.

\section{Summary and conclusions}

A numerical simulation of the ultrasonic propagation in a dental implant is obtained from a model consisting of a single generic implant geometry, which is fully inserted in a jawbone section. The bone is made of cortical and trabecular bone tissues and the implant is fully bonded to the bone. A peri-implant bone layer was assigned various stiffness values and widths to mimic the various stages of osseointegration. The originality of the present paper, compared to (Mathieu et al., 2011a) (Vayron et al., 2015), is first to consider a realistic implant geometry. In addition, compared to (Vayron et al., 2016), the present paper considers i) a combined variation of the Young's modulus, the Poisson's ratio and of the mass density through the parameter $\xi$, ii) a more realistic bone geometry with cortical bone tissue located all around the implant, which is more realistic than what was done in (Vayron et al., 2016) and iii) the influence of the thickness $w$ of the layer where osseointegration occurs, which is an important parameter for the implant success. The characterization is realized with the aid of a new Integral Indicator.

The results show that the weaker the peri-implant layer, the higher the indicator value for all $\mathrm{t}^{*}$. It also appears that the width of deteriorated peri-implant layer has a minor effect on the indicator's values. This fact indicates that the mechanical impedance mismatch between the implant and bone is the main cause for changes in the indicator values. This point is particularly interesting when comparing ultrasonic to resonant frequency measurements. The present results show the dominant contribution of the interfacial wave propagation with respect to the impedance mismatch, which confers a high sensitivity to the "measurements", in contrast with resonant frequency measurements for which the bone+interface+implant is a whole vibrating structure, of which the interface is only a minor component.

The gradients of the indicator values variation with $\xi$ indicate that healing process might be more easily identified at the early stages than at the late stages of bone healing.

Since the differences in the indicator values between a fully healed and fully deteriorated bone are quite large (150-200\%), it is concluded that monitoring US waves reflections can be successfully used to identify peri-implant bone healing. 
Acknowledgement GH was supported by the European Research Council (ERC) under the European Union's Horizon 2020 research and innovation program (grant agreement No 682001, project ERC Consolidator Grant 2015 BoneImplant).

The authors declare they have no conflict of interests.

\section{References}

Albrektsson, T., 2008. Hard tissue implant interface. Aust. Dent. J. 53, S34-S38. doi:10.1111/j.1834-7819.2008.00039.x

American Society for Testing and, M., American Society for Testing and Materials, 2013. Standard Specification for Wrought Titanium-6Aluminum-4Vanadium ELI (Extra Low Interstitial) Alloy for Surgical Implant Applications (UNS R56401).

Aparicio, C., Lang, N.P., Rangert, B., 2006. Validity and clinical significance of biomechanical testing of implant/bone interface. Clin. Oral Implants Res. 17, 2-7. doi:10.1111/j.16000501.2006.01365.x

Atieh, M.A., Alsabeeha, N.H.M., Payne, A.G.T., de Silva, R.K., Schwass, D.S., Duncan, W.J., 2014. The prognostic accuracy of resonance frequency analysis in predicting failure risk of immediately restored implants. Clin. Oral Implants Res. 25, 29-35. doi:10.1111/clr.12057

Atieh, M.A.,Humood Alsabeeha, N., Payne, A.G.T., 2012. Can Resonance Frequency Analysis Predict Failure Risk of Immediately Loaded Implants? The International Journal of Prosthodontics, 326-339

De Almeida, M., Maciel, C., Pereira, J., 2007. Proposal for an Ultrasonic Tool to Monitor the Osseointegration of Dental Implants. Sensors 7, 1224-1237. doi:10.3390/s7071224

Dorogoy, A., Rittel, D., Shemtov-Yona, K., Korabi, R., 2017. Modeling dental implant insertion. J. Mech. Behav. Biomed. Mater. 68, 42-50. doi:10.1016/j.jmbbm.2017.01.021

Drozdz, M.B., 2008. EFFICIENT FINITE ELEMENT MODELLING OF ULTRASOUND WAVES IN ELASTIC MEDIA.

Egerton, J.S., Lowe, M.J.S., Huthwaite, P., Halai, H. V., 2017. A multiband approach for accurate numerical simulation of frequency dependent ultrasonic wave propagation in the 
time domain. J. Acoust. Soc. Am. 142, 1270-1280. doi:10.1121/1.5000492

Gao, X., Fraulob, M., Haïat, G., 2019. Biomechanical behaviours of the bone-implant interface: a review. J. R. Soc. Interface 16, 20190259. doi:10.1098/rsif.2019.0259

Hériveaux, Y., Nguyen, V.-H., Haïat, G., 2018. Reflection of an ultrasonic wave on the boneimplant interface: A numerical study of the effect of the multiscale roughness. J. Acoust. Soc. Am. 144, 488-499. doi:10.1121/1.5046524

Manresa, C., Bosch, M., Echeverría, J.J., 2014. The comparison between implant stability quotient and bone-implant contact revisited: an experiment in Beagle dog. Clin. Oral Implants Res. 25, 1213-1221. doi:10.1111/clr.12256

Mathieu, V., Anagnostou, F., Soffer, E., Haiat, G., 2011a. Numerical simulation of ultrasonic wave propagation for the evaluation of dental implant biomechanical stability. Cit. J. Acoust. Soc. Am. 129, 4062. doi:10.1121/1.3586788

Mathieu, V., Anagnostou, F., Soffer, E., Haïat, G., 2011b. Ultrasonic Evaluation of Dental Implant Biomechanical Stability: An In Vitro Study. Ultrasound Med. Biol. 37, 262-270. doi:10.1016/J.ULTRASMEDBIO.2010.10.008

Mathieu, V., Vayron, R., Soffer, E., Anagnostou, F., Haïat, G., 2012. Influence of Healing Time on the Ultrasonic Response of the Bone-Implant Interface. Ultrasound Med. Biol. 38, 611618. doi:10.1016/J.ULTRASMEDBIO.2011.12.014

Nkenke, E., Hahn, M., Weinzierl, K., Radespiel-Troger, M., Neukam, F.W., Engelke, K., 2003. Implant stability and histomorphometry: a correlation study in human cadavers using stepped cylinder implants. Clin. Oral Implants Res. 14, 601-609. doi:10.1034/j.16000501.2003.00937.x

Rittel, D., Dorogoy, A., Shemtov-Yona, K., 2017. Modelling dental implant extraction by pullout and torque procedures. J. Mech. Behav. Biomed. Mater. 71, 416-427. doi:10.1016/j.jmbbm.2017.04.010

Vayron, R., Karasinski, P., Mathieu, V., Michel, A., Loriot, D., Richard, G., Lambert, G., Haiat, G., 2013. Variation of the ultrasonic response of a dental implant embedded in tricalcium silicate-based cement under cyclic loading. J. Biomech. 46, 1162-1168. doi:10.1016/J.JBIOMECH.2013.01.003

Vayron, R., Mathieu, V., Michel, A., Haïat, G., 2014a. Assessment of In Vitro Dental Implant Primary Stability Using an Ultrasonic Method. Ultrasound Med. Biol. 40, 2885-2894. doi:10.1016/J.ULTRASMEDBIO.2014.03.035

Vayron, R., Nguyen, V.-H., Bosc, R., Naili, S., Haïat, G., 2016. Assessment of the biomechanical stability of a dental implant with quantitative ultrasound: A threedimensional finite element study. J. Acoust. Soc. Am. 139, 773-780. doi:10.1121/1.4941452

Vayron, R., Nguyen, V.-H., Bosc, R., Naili, S., Haïat, G., 2015. Finite element simulation of ultrasonic wave propagation in a dental implant for biomechanical stability assessment. Biomech. Model. Mechanobiol. 14, 1021-1032. doi:10.1007/s10237-015-0651-7

Vayron, R., Nguyen, V.-H., Lecuelle, B., Haiat, G., 2018a. Evaluation of dental implant stability in bone phantoms: Comparison between a quantitative ultrasound technique and resonance frequency analysis. Clin. Implant Dent. Relat. Res. doi:10.1111/cid.12622 
Vayron, R., Nguyen, V., Lecuelle, B., Lomami, H.A., Meningaud, J., Bosc, R., Haiat, G., 2018 b. Comparison of Resonance Frequency Analysis and of Quantitative Ultrasound to Assess Dental Implant Osseointegration. Sensors 18, doi:10.3390/s18051397. doi:10.3390/s18051397

Vayron, R., Soffer, E., Anagnostou, F., Haïat, G., 2014b. Ultrasonic evaluation of dental implant osseointegration. J. Biomech. 47, 3562-3568. doi:10.1016/J.JBIOMECH.2014.07.011

von Wilmowsky, C., Moest, T., Nkenke, E., Stelzle, F., Andreas Schlegel, K., n.d. Implants in bone: Part II. Research on implant osseointegration Material testing, mechanical testing, imaging and histoanalytical methods. doi:10.1007/s10006-013-0397-2

Zanetti, E.M., Pascoletti, G., Calì, M., Bignardi, C., Franceschini, G., 2018. Clinical Assessment of Dental Implant Stability During Follow-Up: What Is Actually Measured, and Perspectives. Biosensors 8, 68. doi:10.3390/bios8030068 